\title{
Rendimiento del talento humano en el ámbito educativo del sur de Manabí a través de la gestión administrativa
}

\author{
Performance of human talent in the educational field of southern Manabi \\ through administrative management
}

Maribel Celi Vásquez Paucar ${ }^{1}$, Zoe Luisa Rodríguez Cotilla ${ }^{2}$, Mariana de Lourdes Cantos Figueroa $^{3} \&$ Teresa Yanina Cañarte Quimis ${ }^{4}$

Recibido: 10-07-2020 / Revisado: 19-08-2020 / Aceptado: 07-09-2020 / Publicado: 03-10-2020

\begin{abstract}
.
DOI: $\underline{\text { https://doi.org/10.33262/cienciadigital.v4i4.1420 }}$

Introduction. Human talent is and has been the significant, efficient, effective and effective resource that an educational institution has, it is the one that manages the activities proposed to achieve the proposed objectives and goals. These training entities, insert into their roles instructed and trained people in the various fields of knowledge, so that together with the educational community they commit to social change. The entities in their management model present fundamental pillars for their development and within them is administrative management, which allows the human being to carry out the documentary process to fulfill the activities and strategies established in the plans presented periodically, it also recognizes the level of knowledge, attitude, skills and competitiveness that the human being possesses, in order to achieve results for the benefit of the institution, therefore the following Objective to transform the performance of human talent in the educational field of southern Manabí through administrative management, the following methodology was considered for its development: The research design was non-experimental, descriptive, qualitative model, type of analytical and exploratory order. The chosen population was 57 teachers, who work with 1,100 students who attend the regular

\footnotetext{
${ }^{1}$ Universidad Estatal del Sur de Manabí, Carrera de Enfermería, Facultad Ciencias de la Salud, Jipijapa, Manabí, Ecuador, maribel.vasquez@unesum.edu.ec

2 Universidad de La Habana Cuba, Facultad de Economía, La Habana, Cuba. zoe@fec.uh.cu

${ }^{3}$ Universidad Estatal del Sur de Manabí, Facultad de Economía, Jipijapa, Manabí, Ecuador. mariana.cantos@unesum.edu.ec

${ }^{4}$ Universidad Estatal del Sur de Manabí, Facultad de Economía, Jipijapa, Manabí, Ecuador. teresa.cañarte@unesum.edu.ec
} 
leveling course at the State University of the South of Manabí. It was concluded with the updating of knowledge in various scientific and technical branches, for which it is described: Development of curricular programming as a strengthening tool, training for teachers, concerning pedagogy and formative creation; administrative management; abilities and skills for the creation and improvement of skills in the teaching - learning process, supported by the creative education guide.

Keywords: Innovation, Training, Teachers, Administration, Leadership, Education.

\section{Resumen.}

Introducción. El talento humano es y ha sido el recurso significativo, eficiente, eficaz y efectivo con el que cuenta una institución educativa, es aquel que gestiona las actividades planteadas para lograr objetivos y metas propuestas. Estas entidades formativas, insertan en sus roles a personas instruidas y capacitadas en los diversos campos de saberes, para que junto a la comunidad educativa se comprometan al cambio social. Las entidades en su modelo de gestión presentan pilares fundamentales para su desarrollo y dentro de ellas se encuentra la gestión administrativa, la que permite al ser humano efectuar el proceso documental para cumplimiento de actividades y estrategias establecidas en las planificaciones presentadas de forma periódica, también reconoce el nivel de conocimiento, actitud, destrezas y competitividad que posee el ser humano, para alcance de resultados en beneficio de la institución, por ello se planteó el siguiente Objetivo, transformar el rendimiento del talento humano en el ambito educativo del sur de Manabí a través de la gestión administrativa, para su desarrollo se consideró la siguiente metodología: El diseño de la investigación fue no experimental, de corte descriptivo, modelo cualitativo, tipo de orden analítico y exploratorio. La población escogida fue de 57 docentes, los cuales trabajan con 1100 estudiantes que asisten al curso regular de nivelación de la Universidad Estatal del Sur de Manabí. Se concluyó con la actualización de conocimientos en diversas ramas científicos y técnicas, para lo que se describe: Desarrollo de programación curricular como herramienta de fortalecimiento, adiestramientos a los docentes, concernientes a la pedagogía y creación formativa; gestión administrativa; habilidades y destrezas para la creación y perfeccionamiento de las habilidades en el proceso enseñanza - aprendizaje, respaldado por la guía de educación creadora.

Palabras claves: Innovación, Capacitación, Docentes, Administración, Liderazgo, Educación.

\section{Introducción.}

La globalización ha traído diversos cambios en el ámbito político, social, educativo y económico, razón por la cual el recurso humano se esfuerza cada día más por generar cambios transcendentales en las instituciones que laboran, sin embargo, no se logra el ansiado 
desarrollo. A nivel mundial existen diversos modelos de gestión formativa que describen políticas, estrategias, metas, acciones y objetivos que conciben mejorar la educación a través del desempeño laboral, pero estudios realizados demuestran que existe un alejamiento entre la gestión educativa y la gestión administrativa, que no permite relacionarse adecuadamente para fortalecer las instituciones (Vásquez, 2020, citado en Ospina 2017).

El fortalecimiento se emprende con el proceso de innovaciones en las empresas, deben realizarse aplicando la planeación, organización, dirección y control de las actividades a perfeccionar y para ello debe estar involucrado y comprometido el talento humano en su totalidad. La elaboración de una planeación tiene como meta, proporcionar una guía para el logro de los objetivos y estrategias institucionales, teniendo en consideración las necesidades de la sociedad a nivel local, nacional e internacional. Para la correcta elaboración del diseño y desarrollo de la planificación se deben considerar aspectos tales como: Análisis y valoración del entorno; Determinación del nivel de desempeño, eficiencia y eficacia de los recursos; Estudio y establecimiento de dificultades organizacionales; Cultura de comunicación efectiva, Construir y socializar visión para el futuro; Desplegar y aplicar estrategias; Inspeccionar y Dirigir los cambios. (Vásquez, 2020, citado en Hellriegel. 2005)

Para aplicar la mejora continua, las organizaciones tendrán en consideración, que el talento humano tiene un dispositivo programado para cumplir con sus objetivos y las organizaciones siguen ese sistema. En este sentido las instituciones sitúan a las personas de acuerdo a sus capacidades y competencias, además permiten que se pronuncien libremente y que cada individuo entienda lo que dice el otro. En el ámbito del desempeño laboral, las personas trabajan por beneficios financieros y progreso propio, dando lugar a las motivaciones, liderazgo y perseverancia para trabajar con pertinencia y responsabilidad (Maldonado, 2017)

$\mathrm{Al}$ respecto (Garbazo, 2016) refiere que los altos dirigentes y jefes de primera línea, personas eficientes, conocen los tiempos adecuados en los que se debe realizar cambios y transformaciones a nivel interno y externo, estos se presentan por la influencia del entorno existente dentro y fuera de la entidad, debido al cambio social, creado por la globalización e internacionalización, lo que conlleva al intercambio de opinión en lo referente a buenas practicas aplicadas en cada institución.

En las entidades educativas se generar innovaciones con el propósito de mejorar el rendimiento y la productividad de sus recursos, tomando como base los documentos rectores para la buena marcha de la organización (Hellriegel, 2005). Por otra parte, la importancia de la gestión administrativa en el progreso institucional, radica en la comprensión total de sus procesos, lo que permite ser proactivo, visionario y eficaz en la dirección de la organización, sin importar su tamaño o capital invertido, por ello es preciso, realizar de forma periódica evaluaciones y de acuerdo a sus resultados, generar planes de acción que apliquen estrategias para obtener ganancias satisfactorias (Vásquez, 2020, citado en Ramírez 2017). 
Para las instituciones el recurso humano es el elemento primordial e indispensable, para su operatividad, por lo que se puede testificar que, en el sistema educativo, el docente, como sujeto dinámico y garante del proceso, cumple con un rol protagónico y de gran compromiso, para lo que debe estar altamente capacitado e instruido en la parte técnica y científica, de cada uno de los ámbitos en los que se despliegan las interacciones con docentes, estudiantes, autoridades y sociedad en general. El talento humano motivado es de significativa y valiosa ayuda para entregar calidad y calidez al usuario (Vásquez, 2020, citado en Vallejo 2017).

En el apoyo a optimizar la calidad de vida del ser humano, la Organización de las Naciones Unidas para la Educación, la Ciencia y la Cultura, dispone de un mandato para abarcar los aspectos de la educación y se apoya en el objetivo de Desarrollo sostenible 4, el que enuncia dentro de sus metas, cumplir con el incremento de las habilidades para un trabajo decoroso y fomentar el emprendimiento, cumpliendo con entornos de enseñanza aprendizaje eficaces, y aumentando significativamente el número de talento humano calificado. (UNESCO, 2019), así también, de acuerdo a la estrategia de educación (2019 - 2030) presentada por el Fondo de Naciones Unidas para la infancia y como cumplimiento a los Objetivos de Desarrollo Sostenibles a nivel mundial, propone el objetivo de proporcionar una visión y un marco estratégico para las preferencias de UNICEF y ODS (UNICEF, 2019).

Con esta manifestación, las instituciones de Educación operatividad su planificación y organización en beneficio del Talento Humano, y Ecuador no es la excepción. En el año 2016 la Universidad Estatal del Sur de Manabí, ubicada en el Cantón Jipijapa, ingresa como socia de un importante proyecto en el que participan 16 universidades Latinoamericanas y 6 Europeas. El objetivo es cambiar el accionar del docente y el tema "De la tradición a la innovación en la formación del profesorado" siglas en Ingles "TO INN". En el proyecto se propone desarrollar un programa integral, basado en cuatro componentes: Política y Gobernanza, dimensión social, pertinencia curricular e innovación docente. Los Resultados a obtener son docentes altamente capacitados en el ámbito pedagógico y administrativo, promover el intercambio de experiencias, fortalecer las buenas practicas que permiten el incremento de la calidad educativa (Montané 2018).

En este andamiaje de metas, la UNESUM, emprende hacia la ejecución de lo planificado, coordinando con directivos y docentes de Nivelación, Director del Distrito de Educación 13D03, Presidente de la Red de maestros y Rectores de las Unidades Educativas de Jipijapa, la propuesta es capacitar al talento humano de acuerdo a la actividad que desempeñan, esto es directivos, administrativos, docentes y personal de servicio, se efectuaron cuatro etapas de capacitación: Didáctica e innovación educativa; Estrategias para la innovación didáctica. Mejora de las prácticas en educación sustentado en el modelo pedagógico constructivista; Elementos de estadística básica en función de la investigación científica en el proceso de nivelación de carreras; Planificación micro curricular - administración y gestión. Inicia su 
plan piloto con 57 docentes de nivelación de la Universidad, la experiencia se basa en la formación e innovación del talento humano a través de la capacitación, sumando para ello un clima y cultura organizacional adecuada que permita al ser humano, tener libertad de expresión y entusiasmo en aprender nuevas pautas para el cumplimiento de sus labores.

El personal académico y Técnico, fue seleccionado analizando cada área de trabajo, la capacitación se impartió en aulas universitarias, la asistencia de carácter presencial y el tiempo de cumplimiento fue 142 horas presenciales y 74 horas no presenciales ( Cevallos, 2019 pp 58-63)

\section{Metodologia.}

El diseño utilizado para la investigación fue no experimental, de corte descriptivo, modelo cualitativo, tipo de orden analítico y exploratorio, porque estudian el comportamiento humano. En la parte explorativa se coordinaron y ejecutaron reuniones con líderes y directivos para determinar las necesidades de cada grupo, en lo descriptivo se fundamenta el tema y objetivo con información recopilada en información bibliográfica y estudio de campo, modelo cualitativo se aplica en determinar grupos a quienes se les impartirá la capacitación. (Hernández, 1997). Con lo que se dio inicio al plan piloto, con la asistencia de 57 docentes clases presenciales, en horario de 19:00 a 22:00, agenda que no interrumpe la labor diaria del docente de la UNESUM.

La planificación de capacitaciones se ejecuta en los años 2028 y 2019, el trabajo desplegado considera horas de teoría y horas de prácticas, cada tema implementa técnicas y estrategias adecuadas para lograr un aprendizaje significativo.

Se lleva un control pormenorizado de las actividades desplegadas, posteriormente se evalúa a cada participante para determinar los cambios generados con el aprendizaje en la capacitación.

La puesta en marcha del plan toma como base el modelo Educativo de la UNESUM el que se sustenta en principios teóricos de carácter humanista, multifuncional, sistémico e histórico, y se despliega de acuerdo a las etapas que se describen a continuación:

\section{Etapa I: Didáctica e innovación educativa.}

a) Reproducción: Competencias referentes a conceptualizaciones básicas de las materias de ejes centrales.

b) Aplicación: Análisis de relaciones conceptuales, reflexión de la realidad donde se aplican.

c) Creación: Destrezas y habilidades para identificar y resolver problemas, determinar el contexto en el que se desarrolla, justificar por qué existe el problema y presentar soluciones de mejoras. 
La medición del rendimiento de los alumnos, debe considerar los tres parámetros reproducción, aplicación y creación. Es necesario determinar los conocimientos previos y habilidades que poseen los participantes.

Valoraciones de la Evaluación:

Responde más del $60 \%$ de las preguntas se considera primer nivel.

Responde más del 50\% de las preguntas se considera segundo nivel.

Responde más del $40 \%$ de las preguntas se considera tercer nivel.

Consideraciones a tener en cuenta para la evaluación.

Desarrollo de talleres de cada clase.

Evolución de conocimientos.

Recursos de la formación (vídeos y plataforma Moodle)

Etapa II. Estrategias para la innovación didáctica. Mejora de las prácticas en educación sustentado en el modelo pedagógico constructivista.

Rol del docente

Como delegado, organizador, regulador, facilitador, debe:

$>$ Establecer un clima organizacional que fomente el trabajo en equipo.

$>$ Indagar sobre las expectativas y objetivos de los, aplicando la psicología

$>$ Determinar instrucciones anteriores de cada estudiante

$>$ Indagar en lo referente al apoyo de sus: familiares, autoridades y maestros

$>$ Contextualizar las acciones.

Rol del discente (Protagonista activo en la construcción del aprendizaje)

$>$ El discente crea documentos informativos aplicando técnicas de estudios y vinculando la teoría con la realidad.

$>$ Participa en eventos académicos científicos y técnicos, de carácter nacional e internacional.

$>$ Propone soluciones a problemas presentados por sus compañeros, debate conceptos y criterios fundamentando su accionar en la práctica y experiencia sobre el tema.

Relación docente - discente:

$>$ El docente ve al discente como una persona digna de respeto y consideración, con capacidad de pensar y con potencial ilimitado para el cambio. 
$>$ Impulsar el desarrollo de estudios que conlleven al cambio de la sociedad, mediante la presentación y ejecución de proyectos nacionales o internacionales.

$>$ Establecer una comunicación efectiva

$>$ Concepción de la evaluación educativa, de diagnóstico, desarrolladora, formativa y de control, basada en: estudios de casos, solución de problemas, proyectos de investigación.

$>$ Determinar el grado de aprendizaje logrado

$>$ Valorar el medio en el que se desarrolla el aprendizaje

Recursos utilizados en la formación (vídeos, Moodle, doodles...)

Al terminar el curso, cada participante presenta un sistema de clases, el que será evaluado por un tribunal y las valoraciones a considerar son: 100 puntos $=2$ puntos; $94=3 ; 88=5 ; 82$ $=4$.

Etapa III. Elementos de estadística básica y descriptiva en función de la investigación científica en el proceso de nivelación de carreras

Contenidos

$>$ Lectura y escritura de datos. Conceptos básicos

$>$ Frecuencia absoluta, relativa

$>$ Frecuencia absoluta acumulada

$>$ Descriptivas numéricas - Media muestra

$>$ Percentiles

$>$ Varianzas

$>$ Ejercicios prácticos

Recursos de la formación (vídeos, Moodle, doodle)

Evaluación. Aplicación de encuesta, criterios de medición - Valoración de 1 a 5 puntos a cada ítem: Tiempo, Colaboración, Metodología, Recursos utilizados

\section{Fase IV. Planificación micro curricular - administración y gestión}

Contenidos:

Elementos Curriculares.

- Evaluación

- Fines

- Recursos

- Objetivos

- Metodología

Administración y Gestión.

- Proceso Administrativo

- Tendencias de Gestión 
- Áreas funcionales

- Calidad de vida en el trabajo del talento humano

- Clima organizacional

- Incentivos y bonificaciones

- Liderazgo

- Emprendimiento

Evaluación: Al terminar el curso, cada participante presenta un sistema de clases, el que será evaluado por un tribunal y las valoraciones a considerar son: 100 puntos $=2$ puntos; $94=3$; $88=5 ; 82=4($ UNESUM, 2016)

\section{Resultados.}

Los resultados obtenidos, son respuestas de los docentes que participaron en el plan de capacitación, tal es el caso que en la tabla 1, se determinan las insuficiencias del grupo de docentes y para ello piden continuidad del sistema de capacitación. Sin embargo, se pudo identificar que la realidad educativa local (tabla 1) manifiesta más del 50\% de los docentes no presentan perfil pedagógico y constituye un reto la implementación de nuevas vías de innovación acorde a las necesidades del área de influencia del proyecto ya que la realidad educativa actual, con la aparición de nuevos sujetos de la educación, estudiantes adultos buscan una oportunidad, y poseen diferentes modos de apropiación de los conocimientos, estudiantes que trabajan, requiere de metodologías y estrategias innovadoras para garantizar que el contenido que llevamos al aula tenga significación social y utilidad práctica.

Se pudo demostrar a pesar de las limitantes en el proceso de innovación, que los diferentes temas que se abordan son pertinentes y están siendo replicados por los docentes universitarios a las instituciones educativas del territorio con gran acogida, así como, las dimensiones y objetivos explicitados por el proyecto son adecuados y contextualizados por los capacitadores para llevarlos a los docentes y estudiantes. (Cevallos, 2019 pp 58-63)

Tabla 1. Resultados de la implementación de la etapa I

Valoración general de la formación

A - Valoración de El tiempo de capacitación fue muy corto, por lo que la solicitud es

la duración continuar con el sistema de preparación, con énfasis en la innovación educativa.

B - Valoración de La totalidad de los participantes mencionan que el ciclo de la participación capacitaciones ha sido provechoso porque innova los conocimientos de cada docente y esto mejora el desempeño laboral. 
C-Valoración de

los formadores

D - Valoración de la metodología

E - Valoración de

los recursos

F-Otros aspectos

a valorar

Adecuación de la

formación a los

objetivos del
Cuentan con mucha experticia y aplicación de métodos y técnicas innovadoras para motivar y lograr un trabajo eficiente y eficaz.

La metodología aplicada, es muy didáctica e invita a reflexionar y querer aprender más sobre los temas impartidos en clases.

Humanos, tecnológicos, de infraestructura, de gestión, están calificados como excelentes

Las temáticas impartidas tributan al estudio de la realidad en que vivimos.

El cumplimiento de etapas y objetivos es satisfactorio, los capacitadores son cumplidos, dinámicos y visionarios. proyecto TO-INN

Fuente: Elaboración propia.

La realización del curso dentro del sistema de la ejecución del proyecto en el área de estudio, se realizó en los meses de junio-agosto del 2018 con 36 horas presenciales y 19 de trabajo autónomo con un $100 \%$ de aceptación de los temas tratados (tabla 2). Se consideró muy útil la ejemplificación de métodos y técnicas que desde el constructivismo se pueden utilizar en el desarrollo del proceso de enseñanza-aprendizaje.

Los diferentes temas que se abordan son pertinentes y están siendo replicados con los docentes universitarios y de las instituciones educativas del territorio con gran acogida. Además, se establecieron coordinaciones con docentes de la Universidad Nacional de Educación (UNAE) para replicar estos temas en el territorio con más de 1380 docentes de los diferentes niveles, considerando que son suficientes y muy útiles las conferencias, y al igual que en los demás temas, las dimensiones y objetivos explicitados por el proyecto son adecuados y contextualizados por los capacitadores para llevarlos a los docentes y estudiantes.

Tabla 2. Resultados de la implementación de la Fase II

\begin{tabular}{cl}
\hline \multicolumn{1}{c}{ Valoración general de la formación } \\
\hline $\begin{array}{c}\text { A - Valoración } \\
\text { de la duración }\end{array}$ & $\begin{array}{l}\text { El curso se desarrolló entre junio-agosto de 2018 con 36 horas } \\
\text { presenciales y 19 de trabajo autónomo (no presenciales), en } \\
\text { general se consideró suficiente la duración. }\end{array}$ \\
$\begin{array}{c}\text { B - Valoración de } \\
\text { la participación }\end{array}$ & $\begin{array}{l}\text { El 100\% de los participantes en encuestas aplicadas declararon } \\
\text { que temas tratados son pertinentes. }\end{array}$ \\
\hline
\end{tabular}


C - Valoración de Es decisivo continuar estas capacitaciones teniendo en cuenta las las formadoras carencias pedagógicas y didácticas de los docentes.

D - Valoración de la metodología Se considera muy útil la ejemplificación de métodos y técnicas que desde el constructivismo se pueden utilizar en el desarrollo del proceso de enseñanza-aprendizaje.

E - Valoración de los recursos

Suficientes y muy útiles las conferencias y materiales entregados Los diferentes temas que se abordan son pertinentes y están siendo replicados por los docentes universitarios a las instituciones $\mathrm{F}$-Otros educativas del territorio con gran acogida. Establecimos aspectos a valorar coordinaciones con docentes de la Universidad Nacional de Educación (UNAE) para replicar estos temas en el territorio con más de 1380 docentes de los diferentes niveles.

Adecuación de la formación a los objeticos de del proyecto TO-INN

Al igual que en los demás temas, las fases y objetivos explicitados son adecuados y contextualizados por los capacitadores para llevarlos a los docentes y estudiantes.

Fuente: Elaboración propia.

Al considerar 36 horas presenciales y 19 de trabajo autónomo se reflexionó en las encuestas aplicadas como generalidad, prolongar la extensión del curso en vigilancia especial para el análisis de casos, donde al existir estimulación por adquirir instrumentos y sapiencias para el progreso incesante, los participantes alcanzaron aplicar las ilustraciones estadísticas relacionados con la producción científica y el $45 \%$ de los docentes participantes lograron publicar sus resultados científicos en revistas indexadas, lo que permite enfatizar que la enseñanza organizacional es un asunto de acomodo del ambiente, donde el conocimiento del recorrido institucional, producto de la concordancia con el entorno aprueba la adaptación y sostenibilidad ante la desafiante sociedad (Garbazo 2016).

Aunque, algunos participantes, a partir del diagnóstico inicial consideraron, poco probable los resultados que finalmente se lograron, en tanto consideran factible el desarrollo de cursos que a partir de las potencialidades de los participantes enaltezcan la motivación para que los involucrados aporten sus iniciativas, y la metodología aplicada estuvo en correspondencia con las necesidades de los participantes.

Al respecto Acosta, (2011), Refiere que se originan culturas y climas organizacionales, producto de la jornada laboral en la resolución de los diferentes inconvenientes, innovación de rutinas, sistemas, modelos, enfoques y procedimientos que les admiten manifestar en forma apropiada lo que sucede en el entorno. 
Tabla 3. Resultados de la implementación de la Fase III

\section{Valoración general de la formación}

A - Valoración de Al considerar 36 horas presenciales y 19 de trabajo autónomo se la duración reflexionó en las encuestas aplicadas como generalidad continuar la extensión del curso en atención diferenciada para el análisis de casos

B - Valoración de Los participantes lograron aplicar los conocimientos estadísticos la participación relacionados con la producción científica y el $45 \%$ de los docentes participantes lograron publicar sus resultados científicos en revistas indexadas

C - Valoración de las formadoras Los formadores a partir del diagnóstico inicial consideraron poco probable los resultados que finalmente se lograron, en tanto consideran factible el desarrollo de cursos que a partir de las potencialidades de los participantes enaltezcan la motivación para que los involucrados aporten sus iniciativas.

D - Valoración de la metodología La metodología aplicada estuvo en correspondencia con las necesidades de los participantes.

E - Valoración de Se utilizaron los recursos descritos en la innovación educativa los recursos

F - Otros aspectos a valorar

Agradecimientos a las aportaciones por parte de la Universidad

Fuente: Elaboración propia.

La fase IV se realizó dentro de la planificación establecida, su ejecución se llevó a cabo en los meses de junio-agosto del 2019 con 36 horas presenciales y 19 de trabajo autónomo con un $100 \%$ de aceptación de los temas tratados (tabla 4).

Se consideró muy útil la revisión y análisis de la Planificación Curricular, así como de la administración y modelos de gestión existentes, para el cumplimiento de objetivos institucionales en beneficio del proceso de enseñanza-aprendizaje. Al respecto Munch, (2010), declara que, la gestión es una agilidad necesaria en cualquier empresa, de hecho, es el modo más efectivo para certificar sus competencias.

Los diferentes temas que se abordan son pertinentes y están siendo replicados por los docentes universitarios a la comunidad educativa de las instituciones del territorio con gran acogida, para fortalecer mediante las capacitaciones, la gestión administrativa - educativa, por ser parte importante para la obtención y priorización de recursos humanos, materiales, tecnológicos y económicos financieros. 
Tabla 4. Resultados de la implementación de la Fase IV

\section{Valoración general de la formación}

A - Valoración de

la duración

B - Valoración de

la participación

El curso se desarrolló entre junio-agosto de 2019 con 36 horas

presenciales y 19 de trabajo autónomo (no presenciales), en general se consideró suficiente la duración.

C - Valoración de

las formadoras

El grupo en general, manifiesta que la participación es muy buena

y que se requiere aplicar el sistema de capacitación en cascada.

Los formadores cuentan con experiencia, conocen los temas impartidos, tienen el perfil de docentes y técnicos en el área de conocimiento que tienen a su cargo.

D - Valoración de

la metodología

Al tomar como referencia la innovación de documentos reales y con el ello el trabajo en equipo según el puesto que desempeñan, se logra construir un documento consensuado y ajustado a la realidad de los docentes.

E - Valoración de

los recursos

Los recursos utilizados tienen calidad y calidez, lo que permite el aprendizaje significativo.

F- Otros aspectos a valorar

Los diferentes temas que se abordan son pertinentes y están siendo replicados por los docentes universitarios a las instituciones educativas del territorio con gran acogida.

Adecuación de la formación a los Las actividades ejecutadas se ajustan al cumplimiento del plan y por ende a los objetivos y metas planificadas.

objeticos de del proyecto TO-INN

Fuente: Elaboración propia.

De acuerdo a lo descrito, Campos (2010) declara que la formación es una profesión complicada, que demanda un aprendizaje inquebrantable e incesante y que su productividad se refleja en la gestión de calidad a largo plazo, sin embargo, la costumbre, la cultura y los procesos desarrollados día a día se vuelven rutinarios y limitan a la innovación en cuanto a procedimientos.

\section{Conclusión.}

- La metodología utilizada permitió dar cumplimiento al fin presentado, los profesores demostraron conseguir niveles de desempeño apropiado para construir procesos de ejercicios integradores, desplegaron destrezas pedagogías, preparación de planes y programas asi como estudio de modelos de gestión, que fortifican su productividad en el contexto del trabajo, se renovaron los conocimientos en programación curricular y modelos de gestión como instrumento de fortalecimiento. Se desarrollaron 
capacitaciones al talento humano relacionadas con la didáctica e innovación educativa; estrategias para la innovación didáctica; progreso de las experiencias en adiestramiento universitario, respaldado en el modelo pedagógico constructivista, así como elementos de estadística básica en función de la investigación científica en el proceso de nivelación de carreras que permitieron la publicación de 12 artículos científicos.

- La puesta en marcha de la práctica explicada, tiene como efecto en primera instancia el establecer la necesidad de preparación en numerosas áreas del acontecer pedagógico, y en la fase final, que los docentes capacitados son replicadores en cascada del conocimiento alcanzado, y, para robustecer esta diligencia social se proyectó la continuidad de las instrucciones mediante un Evento exhaustivo de Educación para el Sur de Manabí.

\section{Referencias bibliográficas.}

Acosta, J. (2011). El aprendizaje organizacional como proceso de innovación en nuevas empresas de base tecnológica, un estudio de casos. Ideas, CONCYTEG 6 (74) 959976.

Cevallos, D. Cordero, G. Alcívar, I. (2019). Buenas prácticas en Educación Superior, Edición: Instituto de Creatividad e Innovación Educativa de la Universidad de valencia 2019. ISBN: 978-84-09-09619-0

Campos, A. (2010). Herramientas para directivos escolares, Editado por Wolters Kluwer España - ISBN: 978 - $84-7197$ - 932 - 2

Chiavenato, I. (2009). Comportamiento organizacional. La dinámica del éxito en las organizaciones - McGraw-Hill Companies, Inc.

Garbazo, G. (2016). Desarrollo organizacional y los procesos de cambio en las instituciones educativas, un reto a la gestión de la educación. Revista Educación enero-junio, 40 (1) $67-87$.

Hellriegel, D., Jackson, S. y Solcum, J. (2005). Administración. Un enfoque basado en competencias (10 Ed.). México: McGraw-Hill -.México.

Hernández, R., Fernández, C. y Baptista, P. (1997). Metodología de la Investigación. McGraw-Hill - Canadá. ISBN: 968 -422-931-3

Maldonado, J. (2017). Comportamiento, desarrollo y cambio organizacional - Genebra.

Montané, A. (2018). Educación Superior y Formación del Profesorado: Gobernanza y política, dimensión social, pertinencia curricular e innovación docente 2018, Edición: Instituto de Creatividad e Innovación Educativa de la Universidad de valencia. Printed in Spain, ISBN: 978-84-09-000784

UNESUM (2016). Modelo educativo de Universidad Estatal del Sur de Manabí año 2016

Munch, L. (2010). Administración, Gestión Organizacional, Enfoques y Proceso Administrativo 2009, respecto a la primera edición en español por PEARSONEDITORES.

Ospina, A.; Burgos, S. y Madera, J. (2017). La gerencia educativa y la gestión al cambio. Diálogos de saberes Na 46, enero-junio de 2017, pp 187-200. 
Rodríguez, A. (2015). Modelo de contextualización del proceso enseñanza-aprendizaje de la asignatura Matemática. Tesis en opción al título de Doctor en Ciencias Pedagógicas. Universidad de Granma. Cuba.

UNICEF (2019). Estrategia de educación del Fondo de Naciones Unidas para la Infancia $2019-2030$

UNESCO (2019) La educación Transforma vidas. Organización de las Naciones Unidas para la Educación, la Ciencia y la Cultura. https://es.unesco.org/node/266395

Vallejo, V; Portalanza, A. (2017). Importancia de la Gestión del Talento Humano como estrategia para la atracción y retención de docentes en las Organizaciones Educativas de Ecuador. PODIUM - Edición especial, abril 2017, pp. 145 - 168 Universidad Espíritu Santo - ISSN: 1390 - 5473

Ramírez, A; Ramírez, R; Calderón, E (2017). La Gestión administrativa en el desarrollo empresarial. Revista: Contribuciones a la Economía, marzo 2017, pp. 145 - 168 ISSN: 1696 - 8360.

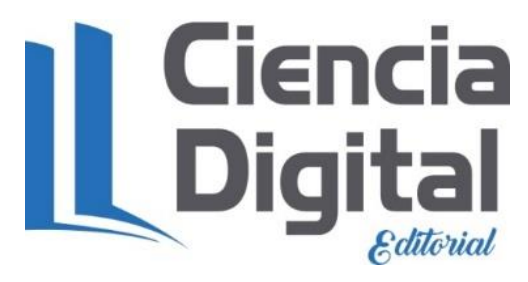




\section{PARA CITAR EL ARTÍCULO INDEXADO.}

Vásquez Paucar, M. C., Rodríguez Cotilla, Z. L., Cantos Figueroa, M. de L., \& Cañarte Quimis, T. Y. (2020). Rendimiento del talento humano en el ámbito educativo del sur de Manabí a través de la gestión administrativa. Ciencia Digital, 4(4), 29-43. https://doi.org/10.33262/cienciadigital.v4i4.1420

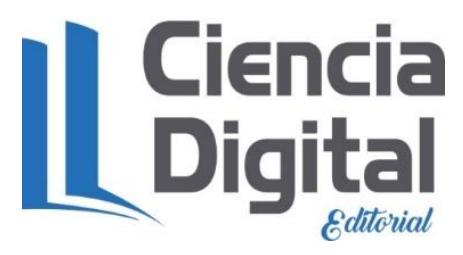

El artículo que se publica es de exclusiva responsabilidad de los autores y no necesariamente reflejan el pensamiento de la Revista Ciencia Digital.

El artículo queda en propiedad de la revista y, por tanto, su publicación parcial y/o total en otro medio tiene que ser autorizado por el director de la Revista Ciencia Digital.
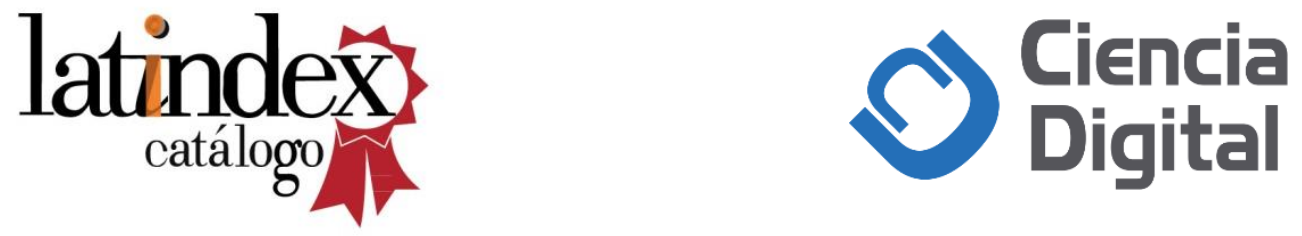